\title{
First-principles calculations of hot-electron lifetimes in metals
}

\author{
I. Campillo \\ Materia Kondentsatuaren Fisika Saila, Zientzi Fakultatea, Euskal Herriko Unibertsitatea, 644 Posta kutxatila, 48080 Bilbo, \\ Basque Country, Spain \\ V. M. Silkin \\ Materialen Fisika Saila, Kimika Fakultatea, Euskal Herriko Unibertsitatea, 1072 Posta kutxatila, 20080 Donostia, \\ Basque Country, Spain \\ J. M. Pitarke \\ Materia Kondentsatuaren Fisika Saila, Zientzi Fakultatea, Euskal Herriko Unibertsitatea, 644 Posta kutxatila, 48080 Bilbo, \\ Basque Country, Spain \\ and Donostia International Physics Center (DIPC) and Centro Mixto CSIC-UPV/EHU, Donostia, Spain \\ E. V. Chulkov \\ Materialen Fisika Saila, Kimika Fakultatea, Euskal Herriko Unibertsitatea, 1072 Posta kutxatila, 20080 Donostia, \\ Basque Country, Spain \\ and Donostia International Physics Center (DIPC) and Centro Mixto CSIC-UPV/EHU, Donostia, Spain \\ A. Rubio \\ Departamento de Física Teórica, Universidad de Valladolid, 47011 Valladolid, Spain \\ P. M. Echenique \\ Materialen Fisika Saila, Kimika Fakultatea, Euskal Herriko Unibertsitatea, 1072 Posta kutxatila, 20080 Donostia, \\ Basque Country, Spain \\ and Donostia International Physics Center (DIPC) and Centro Mixto CSIC-UPV/EHU, Donostia, Spain
}

(Received 3 November 1999)

\begin{abstract}
First-principles calculations of the inelastic lifetime of low-energy electrons in $\mathrm{Al}, \mathrm{Mg}, \mathrm{Be}$, and $\mathrm{Cu}$ are reported. Quasiparticle damping rates are evaluated from the knowledge of the electron self-energy, which we compute within the $G W$ approximation of many-body theory. Inelastic lifetimes are then obtained along various directions of the electron wave vector, with full inclusion of the band structure of the solid. Average lifetimes are also reported, as a function of the electron energy. In $\mathrm{Al}$ and $\mathrm{Mg}$, splitting of the band structure over the Fermi level yields electron lifetimes that are smaller than those of electrons in a free-electron gas. Larger lifetimes are found in $\mathrm{Be}$, as a result of the characteristic dip that this material presents in the density of states near the Fermi level. In $\mathrm{Cu}$, a major contribution from $d$ electrons participating in the screening of electron-electron interactions yields electron lifetimes that are well above those of electrons in a free-electron gas with the electron density equal to that of valence $\left(4 s^{1}\right)$ electrons.
\end{abstract}

\section{INTRODUCTION}

Low-energy excited electrons in metals, with energies larger than $\sim 0.5 \mathrm{eV}$ above the Fermi energy, experience strong electron-electron $(e-e)$ scattering processes. Although inelastic lifetimes of these so-called hot electrons have been investigated for many years on the basis of the free-electrongas (FEG) model of the solid, ${ }^{1-10}$ time-resolved two-photon photoemission (TR-2PPE) experiments have shown the key role that band-structure effects may play in the decay mechanism. ${ }^{11-20}$ First-principles calculations of hot-electron lifetimes that fully include the band structure of the solid have been reported only very recently for aluminum and copper. $^{21,22}$ These calculations ${ }^{21}$ show that actual lifetimes are the result of a delicate balance between localization, density of states, screening, and Fermi-surface topology, even in the case of a free-electron-like metal such as aluminum.
In this paper, we report first-principles calculations of inelastic lifetimes of excited electrons in a variety of real metals. We start with free-electron-like trivalent $(\mathrm{Al})$ and divalent $(\mathrm{Mg})$ metals, and then focus on divalent $\mathrm{Be}$ and the role that $d$ electrons play in a noble metal like $\mathrm{Cu}$. First, we expand the one-electron Bloch states in a plane-wave basis, and solve the Kohn-Sham equation of density-functional theory $^{23}$ (DFT) by invoking the local-density approximation (LDA) for exchange and correlation (XC). The electron-ion interaction is described by means of nonlocal, normconserving ionic pseudopotentials, and we use the oneelectron Bloch states to evaluate the screened Coulomb interaction in the random-phase approximation (RPA) ${ }^{24} \mathrm{We}$ finally evaluate the lifetime of an excited Bloch state from the knowledge of the imaginary part of the electron selfenergy, which we compute within the $G W$ approximation of many-body theory. ${ }^{25}$ Our calculations indicate that scattering 
rates may strongly depend, for a given electron energy, on the direction of the wave vector of the initial state. Also, average lifetimes, as obtained by averaging over all wave vectors and bands with the same energy, are found to deviate considerably from those derived for a FEG.

The rest of this paper is organized as follows: Explicit expressions for the electron decay rate in periodic crystals are derived in Sec. II, within the $G W$ approximation of many-body theory. Calculated inelastic lifetimes of hot electrons in $\mathrm{Al}, \mathrm{Mg}, \mathrm{Be}$, and $\mathrm{Cu}$ are presented in Sec. III, and the conclusions are given in Sec. IV. Atomic units are used throughout, i.e., $e^{2}=\hbar=m_{e}=1$.

\section{THEORY}

Take an inhomogeneous electron system. In the framework of many-body theory, ${ }^{24}$ the damping rate $\tau_{i}^{-1}$ of an excited electron in the state $\phi_{i}(\mathbf{r})$ with energy $E_{i}$ is obtained from the knowledge of the imaginary part of the electron self-energy, $\Sigma\left(\mathbf{r}, \mathbf{r}^{\prime} ; E_{i}\right)$, as

$$
\tau_{i}^{-1}=-2 \int \mathrm{d} \mathbf{r} \int \mathrm{d} \mathbf{r}^{\prime} \phi_{i}^{*}(\mathbf{r}) \operatorname{Im} \Sigma\left(\mathbf{r}, \mathbf{r}^{\prime} ; E_{i}\right) \phi_{i}\left(\mathbf{r}^{\prime}\right) .
$$

In the $G W$ approximation, ${ }^{25}$ one considers only the firstorder term in a series expansion of the self-energy in terms of the screened Coulomb interaction:

$$
\Sigma\left(\mathbf{r}, \mathbf{r}^{\prime} ; E_{i}\right)=\frac{i}{2 \pi} \int d E G\left(\mathbf{r}, \mathbf{r}^{\prime} ; E_{i}-E\right) W\left(\mathbf{r}, \mathbf{r}^{\prime} ; E\right),
$$

where $G\left(\mathbf{r}, \mathbf{r}^{\prime} ; E_{i}-E\right)$ represents the one-particle Green function and $W\left(\mathbf{r}, \mathbf{r}^{\prime} ; E\right)$ is the time-ordered screened Coulomb interaction. After replacing the Green function $(G)$ by the zero-order approximation $\left(G^{0}\right)$, the imaginary part of the self-energy can be evaluated explicitly:

$$
\operatorname{Im} \Sigma\left(\mathbf{r}, \mathbf{r}^{\prime} ; E_{i}\right)=\sum_{f} \phi_{f}^{*}\left(\mathbf{r}^{\prime}\right) \operatorname{Im} W\left(\mathbf{r}, \mathbf{r}^{\prime} ; \omega\right) \phi_{f}(\mathbf{r})
$$

where $\omega=E_{i}-E_{f}$ represents the energy transfer, the sum is extended over a complete set of final states $\phi_{f}(\mathbf{r})$ with energy $E_{f}\left(E_{F} \leqslant E_{f} \leqslant E_{i}\right), E_{F}$ is the Fermi energy, and

$$
\begin{aligned}
W\left(\mathbf{r}, \mathbf{r}^{\prime} ; \omega\right)= & v\left(\mathbf{r}-\mathbf{r}^{\prime}\right)+\int \mathrm{d} \mathbf{r}_{1} \int \mathrm{d} \mathbf{r}_{2} v\left(\mathbf{r}-\mathbf{r}_{1}\right) \\
& \times \chi\left(\mathbf{r}_{1}, \mathbf{r}_{2} ; \omega\right) v\left(\mathbf{r}_{2}-\mathbf{r}^{\prime}\right) .
\end{aligned}
$$

Here, $v\left(\mathbf{r}-\mathbf{r}^{\prime}\right)$ represents the bare Coulomb interaction, and $\chi\left(\mathbf{r}, \mathbf{r}^{\prime} ; \omega\right)$ is the density-density correlation function of the solid.

In the framework of time-dependent density-functional theory, ${ }^{26,27}$ the density-density correlation function satisfies the integral equation

$$
\begin{aligned}
\chi\left(\mathbf{r}, \mathbf{r}^{\prime} ; \omega\right)= & \chi^{0}\left(\mathbf{r}, \mathbf{r}^{\prime} ; \omega\right)+\int \mathrm{d} \mathbf{r}_{1} \int \mathrm{d} \mathbf{r}_{2} \chi^{0}\left(\mathbf{r}, \mathbf{r}_{1} ; \omega\right) \\
& \times\left[v\left(\mathbf{r}_{1}-\mathbf{r}_{2}\right)+K^{x c}\left(\mathbf{r}_{1}, \mathbf{r}_{2} ; \omega\right)\right] \chi\left(\mathbf{r}_{2}, \mathbf{r}^{\prime} ; \omega\right),
\end{aligned}
$$

where $\chi^{0}\left(\mathbf{r}, \mathbf{r}^{\prime} ; \omega\right)$ is the density-density correlation function of noninteracting Kohn-Sham electrons, as described by the solutions of the time-dependent counterpart of the KohnSham equation. In usual practice, these amplitudes are approximated by standard LDA wave functions. The kernel $K^{x c}\left(\mathbf{r}_{1}, \mathbf{r}_{2} ; \omega\right)$, which accounts for the reduction in the $e-e$ interaction due to the existence of short-range XC effects, is obtained from the knowledge of the XC energy functional. In the RPA, this kernel is taken to be zero.

For periodic crystals, one may introduce the following Fourier expansion for the screened interaction of Eq. (4):

$$
\begin{aligned}
W\left(\mathbf{r}, \mathbf{r}^{\prime} ; \omega\right)= & \frac{1}{\Omega} \sum_{\mathbf{q}} \sum_{\mathbf{G}, \mathbf{G}^{\prime}} e^{i(\mathbf{q}+\mathbf{G}) \cdot \mathbf{r}} e^{-\mathrm{i}\left(\mathbf{q}+\mathbf{G}^{\prime}\right) \cdot \mathbf{r}^{\prime}} \\
& \times v_{\mathbf{G}}(\mathbf{q}) \boldsymbol{\epsilon}_{\mathbf{G}, \mathbf{G}^{\prime}}^{-1}(\mathbf{q}, \omega),
\end{aligned}
$$

where the first sum is extended over the first Brillouin zone (BZ), $\mathbf{G}$ and $\mathbf{G}^{\prime}$ are reciprocal lattice vectors, $\Omega$ is the normalization volume, $v_{\mathbf{G}}(\mathbf{q})$ represents the Fourier coefficients of the bare Coulomb interaction, and $\epsilon_{\mathbf{G}, \mathbf{G}^{\prime}}^{-1}(\mathbf{q}, \omega)$ are the Fourier coefficients of the inverse dielectric function,

$$
\epsilon_{\mathbf{G}, \mathbf{G}^{\prime}}^{-1}(\mathbf{q}, \omega)=\delta_{\mathbf{G}, \mathbf{G}^{\prime}}+\chi_{\mathbf{G}, \mathbf{G}^{\prime}}(\mathbf{q}, \omega) v_{\mathbf{G}^{\prime}}(\mathbf{q}) .
$$

Within RPA,

$$
\epsilon_{\mathbf{G}, \mathbf{G}^{\prime}}(\mathbf{q}, \omega)=\delta_{\mathbf{G}^{\prime}, \mathbf{G}^{\prime}}-\chi_{\mathbf{G}, \mathbf{G}^{\prime}}^{0}(\mathbf{q}, \omega) v_{\mathbf{G}^{\prime}}(\mathbf{q}),
$$

where $\chi_{\mathbf{G}_{\mathbf{G}} \mathbf{G}^{\prime}}^{0}(\mathbf{q}, \omega)$ are the Fourier coefficients of the densitydensity correlation function of noninteracting Kohn-Sham electrons (see, e.g., Ref. 28).

After introduction of the Fourier representation of Eq. (6) into Eq. (3), and in the limit that the volume of the system $\Omega$ becomes infinite, one finds the following expression for the damping rate of an electron in the state $\phi_{\mathbf{k}, n_{i}}(\mathbf{r})$ with energy $E_{\mathbf{k}, n_{i}}$ :

$$
\begin{aligned}
\tau_{i}^{-1}= & \frac{1}{\pi^{2}} \sum_{f} \int_{\mathrm{BZ}} \operatorname{dq} \underset{\mathbf{q}, \mathbf{G}^{\prime}}{ } \frac{B_{i f}^{*}(\mathbf{q}+\mathbf{G}) B_{i f}\left(\mathbf{q}+\mathbf{G}^{\prime}\right)}{|\mathbf{q}+\mathbf{G}|^{2}} \\
& \times \operatorname{Im}\left[-\epsilon_{\mathbf{G}, \mathbf{G}^{\prime}}^{-1}(\mathbf{q}, \omega)\right],
\end{aligned}
$$

where $\omega=E_{\mathbf{k}, n_{i}}-E_{\mathbf{k}-\mathbf{q}, n_{f}}$ and

$$
B_{i f}(\mathbf{q}+\mathbf{G})=\int \mathrm{d} \mathbf{r} \phi_{\mathbf{k}, n_{i}}^{*}(\mathbf{r}) e^{i(\mathbf{q}+\mathbf{G}) \cdot \mathbf{r}} \phi_{\mathbf{k}-\mathbf{q}, n_{f}}(\mathbf{r}) .
$$

Couplings of the wave vector $\mathbf{q}+\mathbf{G}$ to wave vectors $\mathbf{q}$ $+\mathbf{G}^{\prime}$ with $\mathbf{G} \neq \mathbf{G}^{\prime}$ appear as a consequence of the existence of electron-density variations in real solids. If these terms, representing the so-called crystalline local-field effects, are neglected, one can write

$$
\tau_{i}^{-1}=\frac{1}{\pi^{2}} \sum_{f} \int_{\mathrm{BZ}} \mathrm{d} \mathbf{q} \sum_{\mathbf{G}} \frac{\left|B_{i f}(\mathbf{q}+\mathbf{G})\right|^{2}}{|\mathbf{q}+\mathbf{G}|^{2}} \frac{\operatorname{Im}\left[\epsilon_{\mathbf{G}, \mathbf{G}}(\mathbf{q}, \omega)\right]}{\left|\epsilon_{\mathbf{G}, \mathbf{G}}(\mathbf{q}, \omega)\right|^{2}} .
$$

Within RPA, Eq. (8) yields 


$$
\begin{aligned}
\operatorname{Im}\left[\boldsymbol{\epsilon}_{\mathbf{G}, \mathbf{G}}(\mathbf{q}, \omega)\right]= & \frac{2 \pi v_{\mathbf{G}}(\mathbf{q})}{\Omega} \sum_{\mathbf{k}}^{\mathrm{BZ}} \sum_{n, n^{\prime}}\left(f_{\mathbf{k}, n}-f_{\mathbf{k}+\mathbf{q}, n^{\prime}}\right) \\
& \times\left|\left\langle\phi_{\mathbf{k}, n}\left|e^{-\mathrm{i}(\mathbf{q}+\mathbf{G}) \cdot \mathbf{r}}\right| \phi_{\mathbf{k}+\mathbf{q}, n^{\prime}}\right\rangle\right|^{2} \\
& \times \delta\left(\omega-E_{\mathbf{k}+\mathbf{q}, n^{\prime}}+E_{\mathbf{k}, n}\right) .
\end{aligned}
$$

Hence, the imaginary part of $\epsilon_{\mathbf{G}, \mathbf{G}}(\mathbf{q}, \omega)$ represents a measure of the number of states available for real transitions involving a given momentum and energy transfer $\mathbf{q}+\mathbf{G}$ and $\omega$, respectively, which is renormalized by the coupling between initial and final states. The factor $\left|\epsilon_{\mathbf{G}, \mathbf{G}}(\mathbf{q}, \omega)\right|^{-2}$ in Eq. (11) accounts for the screening in the interaction with the probe electron. Initial and final states of the probe electron enter through the coefficients $B_{i f}(\mathbf{q}+\mathbf{G})$.

If all one-electron Bloch states entering both the coefficients $B_{i f}(\mathbf{q}+\mathbf{G})$ and the dielectric function $\epsilon_{\mathbf{G}, \mathbf{G}^{\prime}}(\mathbf{q}, \omega)$ were represented by plane waves, then Eqs. (9) and (11) would exactly coincide with the $G W$ scattering rate of excited electrons in a FEG, as obtained by Quinn and Ferrell ${ }^{1}$ and by Ritchie. ${ }^{2}$ For hot electrons with energies very near the Fermi level $\left(E_{i} \approx E_{F}\right)$ this result yields, in the high-density limit $\left(r_{s} \ll 1\right),{ }^{29}$ the well-known formula of Quinn and Ferrell, ${ }^{1}$

$$
\tau_{i}^{\mathrm{QF}}=263 r_{s}^{-5 / 2}\left(E_{i}-E_{F}\right)^{-2} \mathrm{eV}^{2} \text { fs. }
$$

For a detailed discussion of the range of validity of this approach, see Ref. 28.

We note that the decay $\tau_{i}^{-1}$ of hot electrons in periodic crystals depends on both the wave vector $\mathbf{k}$ and the band index $n_{i}$ of the initial Bloch state. Nevertheless, we also define $\tau^{-1}(E)$, as the average of $\tau^{-1}(\mathbf{k}, n)$ over all wave vectors and bands lying with the same energy in the irreducible wedge of the Brillouin zone (IBZ). Decay rates of hot electrons lying outside the IBZ are considered by simply using the symmetry property $\tau^{-1}(S \mathbf{k}, n)=\tau^{-1}(\mathbf{k}, n)$, where $S$ represents an operator of the point group of the crystal.

For the evaluation of the polarizability $\chi_{\mathbf{G}, \mathbf{G}^{\prime}}^{0}(\mathbf{q}, \omega)$ and the coefficients $B_{i f}(\mathbf{q}+\mathbf{G})$, Eq. (10), we use the selfconsistent LDA eigenfunctions of the one-electron KohnSham Hamiltonian of DFT, which we first expand in a planewave basis,

$$
\phi_{\mathbf{k}, n}(\mathbf{r})=\frac{1}{\sqrt{\Omega}} \sum_{\mathbf{G}} u_{\mathbf{k}, n}(\mathbf{G}) e^{i(\mathbf{k}+\mathbf{G}) \cdot \mathbf{r}}
$$

The electron-ion interaction is described by means of nonlocal, norm-conserving ionic pseudopotentials, ${ }^{30,31}$ and the $\mathrm{XC}$ potential is obtained in the LDA with use of the Perdew-Zunger ${ }^{32}$ parametrization of the XC energy of Ceperley and Alder. ${ }^{33}$

Well-converged results have been found with the introduction in Eq. (14) of kinetic-energy cutoffs of 12, 6, and 20 $\mathrm{Ry}$ for $\mathrm{Al}, \mathrm{Mg}$, and $\mathrm{Be}$, respectively. In the case of $\mathrm{Cu}$, all $4 s^{1}$ and $3 d^{10}$ Bloch states have been kept as valence electrons in the pseudopotential generation, and an energy cutoff as large as 75 Ry has been required, thereby keeping $~ 900$ plane waves in the expansion of Eq. (14). Though allelectron schemes, such as the full-potential linearized augmented plane-wave method, ${ }^{34}$ are expected to be better suited for the description of the response of localized $d$ elec-

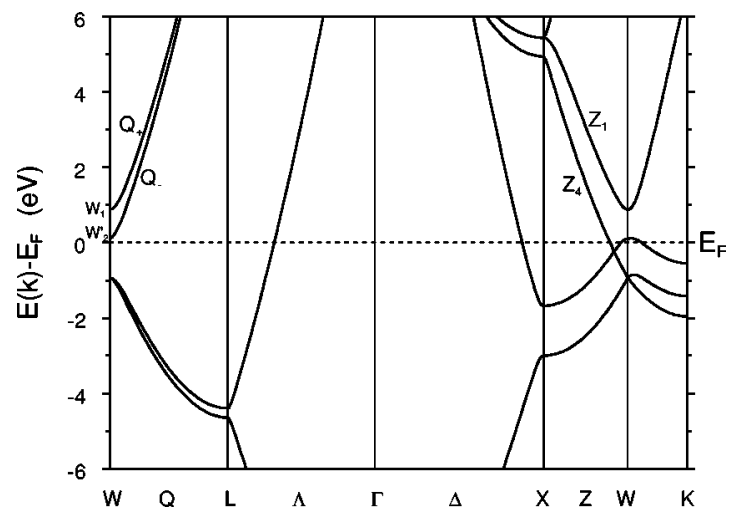

FIG. 1. Calculated band structure of $\mathrm{Al}$ along certain symmetry directions.

trons, the plane-wave pseudopotential approach has already been successfully incorporated in the description of the dynamical response of copper. ${ }^{35}$

Samplings over the BZ required for the evaluation of both the dielectric matrix and the hot-electron decay rate have been performed on Monkhorst-Pack meshes: ${ }^{36}$ $20 \times 20 \times 20$ for $\mathrm{Al}, 24 \times 24 \times 10$ for $\mathrm{Mg}, 24 \times 24 \times 16$ for $\mathrm{Be}$, and $16 \times 16 \times 16$ for $\mathrm{Cu}$. For hot-electron energies under study $\left(E-E_{F} \sim 0.5-4.0 \mathrm{eV}\right)$, the inclusion of up to 40 bands has been required, and the sum in Eq. (11) has been extended over $15 \mathbf{G}$ vectors of the reciprocal lattice, the magnitude of the maximum momentum transfer $\mathbf{q}+\mathbf{G}$ being well over the upper limit of $\sim 2 q_{F}\left(q_{F}\right.$ is the Fermi momentum). For the evaluation of hot-electron lifetimes from Eq. (9), with full inclusion of crystalline local-field effects, dielectric matrices as large as $40 \times 40$ have been considered.

\section{RESULTS AND DISCUSSION}

\section{A. Aluminum}

Due to the free-electron-like nature of the energy bands of face-centered-cubic aluminum (see Fig. 1), a simple metal with no $d$ bands, the impact of the band structure on the electronic excitations had been presumed for many years to be small. However, $\mathrm{x}$-ray measurements ${ }^{37,38}$ and careful firstprinciples calculations of the dynamical density response of this material ${ }^{39,40}$ have shown that band-structure effects cannot be neglected. Full band-structure calculations of the electronic stopping power of $\mathrm{Al}$ for slow ions have shown that the energy loss is $\sim 7 \%$ larger than that of a FEG. ${ }^{41}$ Our present calculations indicate that actual hot-electron lifetimes in $\mathrm{Al}$ are $\sim 35 \%$ smaller than those of electrons in a FEG.

Our ab initio $G W$-RPA calculation of the average lifetime $\tau(E)$ of hot electrons in $\mathrm{Al}$, as obtained from Eq. (9) with full inclusion of crystalline local-field effects, is presented in Fig. 2 by solid circles. The $G W$-RPA lifetime of hot electrons in a FEG with the electron density equal to that of valence electrons in $\mathrm{Al}\left(r_{s}=2.07\right)$ is exhibited in the same figure, by a solid line. Our calculations indicate that the lifetime of hot electrons in $\mathrm{Al}$ is, within RPA, smaller than that of electrons in a FEG with $r_{s}=2.07$ by a factor of $\sim 0.65$. We have performed band-structure calculations of Eq. (9) with and without [see also Eq. (11)] the inclusion of crystalline local-field corrections, and have found that these correc- 


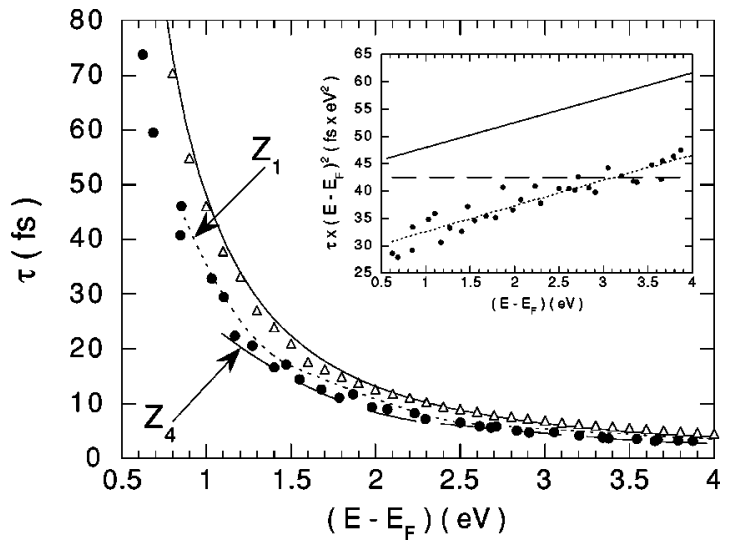

FIG. 2. Hot-electron lifetimes in Al. Solid circles represent our full $a b$ initio calculation of $\tau(E)$, as obtained after averaging $\tau\left(\mathbf{k}, n_{i}\right)$ of either Eq. (9) or Eq. (11) over wave vectors and over the band structure for each $\mathbf{k}$. The solid line represents the lifetime of hot electrons in a FEG with $r_{s}=2.07$, as obtained within the full $G W$-RPA. Open triangles represent the result obtained from Eq. (11) by replacing hot-electron initial and final states in $\mid B_{i f}(\mathbf{q}$ $+\left.\mathbf{G}\right|^{2}$ by plane waves, but with full inclusion of the band structure in the evaluation of $\operatorname{Im}\left[-\epsilon_{\mathbf{G}, \mathbf{G}}^{-1}(\mathbf{q}, \omega)\right]$. Dotted and long-dashed lines represent the lifetime of hot electrons in bands $Z_{1}$ and $Z_{4}$, respectively, with the wave vector along the $W X$ direction. The inset exhibits scaled lifetimes of hot electrons in Al. Solid circles and the solid line represent band-structure and FEG calculations, respectively, both within $G W$-RPA. The dashed line represents the prediction of Eq. (13).

tions are negligible for electron energies under study. This is an expected result, since Al crystal does not present strong density gradients nor special electron density directions (bondings).

In order to understand the origin of band-structure effects on hot-electron lifetimes in $\mathrm{Al}$, we first focus on the role that the band structure plays in the creation of electron-hole $(e-h)$ pairs. Hence, we evaluate hot-electron lifetimes from either Eq. (9) or Eq. (11) by replacing the electron initial and final states in $\left|B_{i f}(\mathbf{q}+\mathbf{G})\right|^{2}$ by plane waves (plane-wave calculation). The result we obtain with full inclusion of the band structure of the crystal in the evaluation of $\operatorname{Im} \epsilon_{\mathbf{G}, \mathbf{G}^{\prime}}^{-1}(\mathbf{q}, \omega)$ is represented in Fig. 2 by open triangles. Due to splitting of the band structure over the Fermi level, new channels are opened for $e-h$ pair production, and band-structure effects tend, therefore, to decrease the lifetime of very-low-energy electrons by $\sim 5 \%$, as in the case of slow ions. ${ }^{41}$

In the case of moving ions, differences between actual decay rates and those obtained in a FEG only enter through the so-called energy-loss matrix, $\operatorname{Im}\left[-\epsilon_{\mathbf{G}, \mathbf{G}^{\prime}}^{-1}(\mathbf{q}, \omega)\right]$. However, hot-electron decay rates are also sensitive to the actual initial and final states entering the coefficients $B_{i f}(\mathbf{q}+\mathbf{G})$. Differences between our full (solid circles) and plane-wave (open triangles) calculations come from this sensitivity of hot-electron initial and final states on the band structure of the crystal, showing that the splitting of the band structure over the Fermi level now plays a major role in lowering the hot-electron lifetime.

Scaled lifetimes, $\tau(E) \times\left(E-E_{F}\right)^{2}$, of hot electrons in $\mathrm{Al}$, as obtained from our full band-structure calculation (solid circles) and from the FEG model with $r_{s}=2.07$ (solid line),

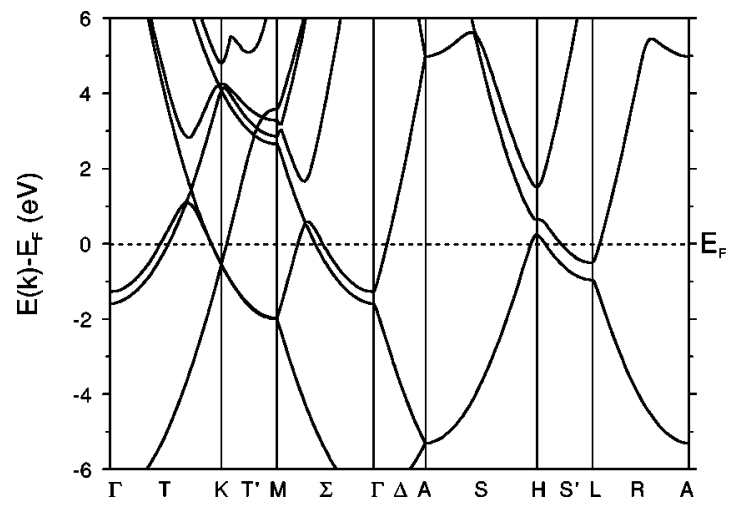

FIG. 3. Calculated band structure of $\mathrm{Mg}$ along certain symmetry directions.

are represented in the inset of Fig. 2. In the limit $E \rightarrow E_{F}$, the available phase space for real transitions is simply $E-E_{F}$, which yields the $\left(E-E_{F}\right)^{-2}$ quadratic scaling of very-lowelectron energies in a FEG, as predicted by Eq. (13) (dashed line). ${ }^{42}$ However, as the energy increases, momentum and energy conservation prevents the available phase space from being as large as $E-E_{F}$, and the lifetime of electrons in a FEG depart, therefore, from the $\left(E-E_{F}\right)^{-2}$ scaling. For energies under study, band-structure effects in $\mathrm{Al}$ are found to be nearly energy independent; hence, our calculated lifetimes are found to approximately scale as in the case of electrons in a FEG, and they slightly depart, therefore, from the $(E$ $\left.-E_{F}\right)^{-2}$ scaling. The agreement at $E-E_{F} \sim 3 \mathrm{eV}$ between actual lifetimes and those predicted by Eq. (13) is simply due to the nearly thorough compensation, at these energies, between the departure of this formula from full free-electrongas RPA calculations and band-structure effects.

Although the energy bands of Al show an overall similarity with the fcc free-electron band structure, in the vicinity of the $W$ point there are large differences between the two cases (see Fig. 1). At this point, the free-electron parabola opens an energy gap around the Fermi level and splits along the $W X$ direction into two bands $\left(Z_{1}\right.$ and $\left.Z_{4}\right)$ with energies over the Fermi level. We have calculated lifetimes of hot electrons in these bands, with the wave vector along the $W X$ direction. The results of these calculations are exhibited in Fig. 2, as a function of energy, by dotted $\left(Z_{1}\right)$ and long-dashed lines $\left(Z_{4}\right)$. Although hot electrons in the $Z_{1}$ band have one more channel available to decay along the $W X$ direction, the band gap at the $W$ point around the Fermi level results in hot electrons living longer on the $Z_{1}$ than on the $Z_{4}$ band. When the hot-electron energy is well above the Fermi level $(E$ $-E_{F}>4 \mathrm{eV}$ ), both $Z_{1}$ and $Z_{4}$ lifetimes nearly coincide with the average values represented by solid circles. We have evaluated hot-electron lifetimes along other directions of the wave vector, and have found that differences between these results and average lifetimes are not larger than those obtained along the $W X$ direction. This is in disagreement with the calculations reported by Schöne et al. ${ }^{22}$ In particular, the bending of the hot-electron lifetime along the $W L$ direction at $\sim 1 \mathrm{eV}$ reported in Ref. 22 is not present in our calculations. The origin of this discrepancy is the crossing near the Fermi level between bands $Q_{+}$and $Q_{-}$along the $W L$ direction reported in Ref. 22. This crossing is absent in the present (see Fig. 1) and previous ${ }^{30,43-45}$ self-consistent band- 


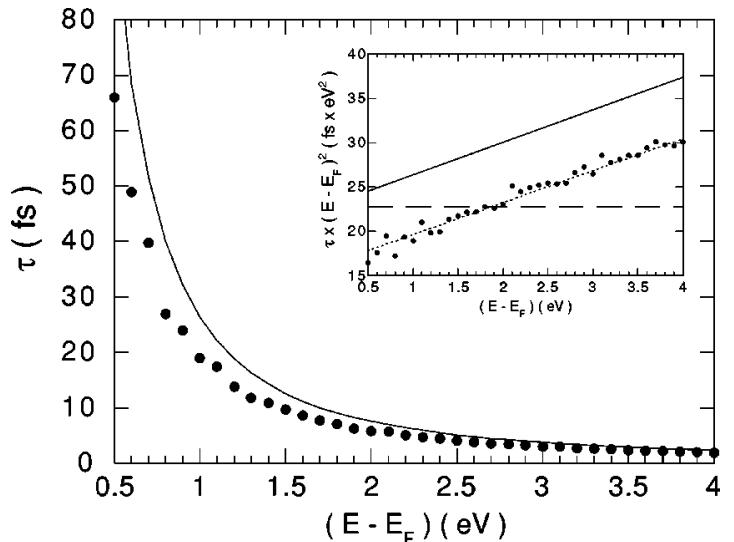

FIG. 4. Hot-electron lifetimes in Mg. As in Fig. 2 with $r_{s}$ $=2.66$.

structure calculations, which all show that at the $W$ point of the $\mathrm{Al}$ band structure the level $W_{2}^{\prime}$ is below $W_{1}$.

\section{B. Magnesium}

In Fig. 3 we show the band structure of hexagonal closedpacked (hcp) magnesium. There is a close resemblance for energies $E<E_{F}$ between this band structure and that of free electrons, though the free-electron parabola now splits near the Fermi level along certain symmetry directions. As a result, the energy-loss function, $\operatorname{Im}\left[-\epsilon_{\mathbf{G}, \mathbf{G}^{\prime}}^{-1}(\mathbf{q}, \omega)\right]$, of this material is approximately well described within a free-electron model, and band-structure effects on hot-electron lifetimes enter mainly, as in the case of $\mathrm{Al}$, through the sensitivity of hot-electron initial and final states on the band structure of the crystal.

Our $a b$ initio calculation of the average lifetime $\tau(E)$ of hot electrons in $\mathrm{Mg}$, as obtained from Eq. (9) with full inclusion of crystalline local-field effects, is presented in Fig. 4 by solid circles, together with the lifetime of hot electrons in a FEG with the electron density equal to that of valence electrons in $\mathrm{Mg}\left(r_{s}=2.66\right)$. As in the case of $\mathrm{Al}$, we have found that local-field corrections are negligible for electron energies under study.

Scaled lifetimes of hot electrons in $\mathrm{Mg}$, as obtained from our full band-structure calculations (solid circles) and from the FEG model with $r_{s}=2.66$ (solid line), are represented in the inset of Fig. 4. We note that actual lifetimes in this material scale with energy approximately as in the case of electrons in a FEG, thereby slightly deviating from the $(E$ $\left.-E_{F}\right)^{-2}$ scaling predicted by Eq. (13) (dashed line). Because of splitting of the band structure over the Fermi level new decay channels are opened, not present in the case of a FEG, and band-structure effects tend, therefore, to decrease the lifetime of hot electrons in $\mathrm{Mg}$ by a factor of $\sim 0.75$. Since the splitting of the band structure of $\mathrm{Mg}$ is not as pronounced as that of $\mathrm{Al}$, the departure of actual lifetimes from those of electrons in a FEG is found to be smaller in $\mathrm{Mg}$ than in $\mathrm{Al}$.

\section{Beryllium}

Among the so-called simple metals, with no $d$ bands, beryllium presents distinctive features in that its band structure

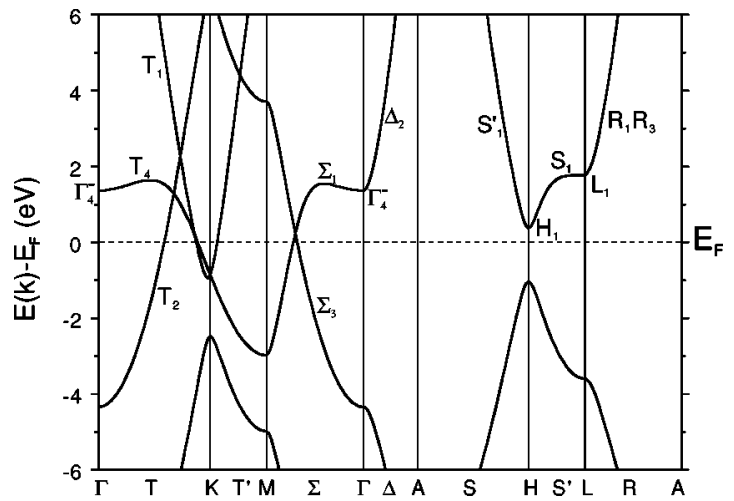

FIG. 5. Calculated band structure of Be along certain symmetry directions.

(see Fig. 5) exhibits the largest departure from free-electron behavior. Both Be and $\mathrm{Mg}$ have hcp crystal structure and two conduction electrons per atom. Nevertheless, the electronic structure of $\mathrm{Be}$ is qualitatively different from that of $\mathrm{Mg}$, the Be band gaps at points $\Gamma, H$, and $L$ being much larger than those in $\mathrm{Mg}$. Also, the Be band gaps are located on both sides of the Fermi level, and the density of states (DOS) of this material falls to a sharp minimum near the Fermi level.

Our band-structure calculation of the average lifetime $\tau(E)$ of hot electrons in Be is shown in Fig. 6 by solid circles, as obtained from Eq. (9). The lifetime of hot electrons in a FEG with the electron density equal to that of valence electrons in $\mathrm{Be}\left(r_{s}=1.87\right)$ is represented in the same figure by a solid line. In the inset, the corresponding scaled calculations are plotted, together with the results obtained from Eq. (13) (dashed line). It can be seen that large deviations from the FEG calculation occur for electron energies near the Fermi level $\left(E-E_{F}<3 \mathrm{eV}\right)$, especially at $E-E_{F}$ $\sim 1.4$ and $1.8 \mathrm{eV}$ where the presence of band gaps at points $\Gamma$ and $L$ plays a key role. We note that the deep departure from free-electron behavior of the beryllium DOS near the Fermi level tends to increase the inelastic lifetime of all excited Bloch states. Furthermore, actual lifetimes strongly deviate from the $\sim\left(E-E_{F}\right)^{-2}$ scaling predicted within Fermi-liquid theory. This deviation comes from the contribution to the average lifetime due to Bloch states near the points $\Gamma$ and $L$ with energies close to the energy gap.

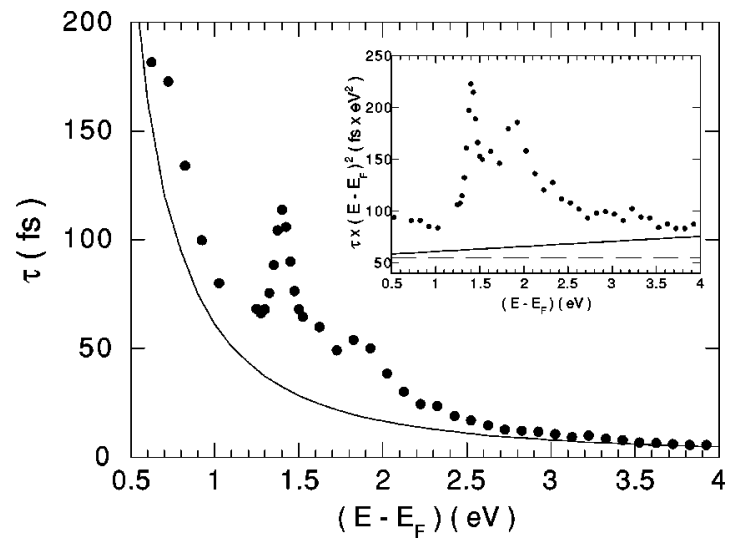

FIG. 6. Hot-electron lifetimes in Be. As in Fig. 2 with $r_{s}$ $=1.87$. 


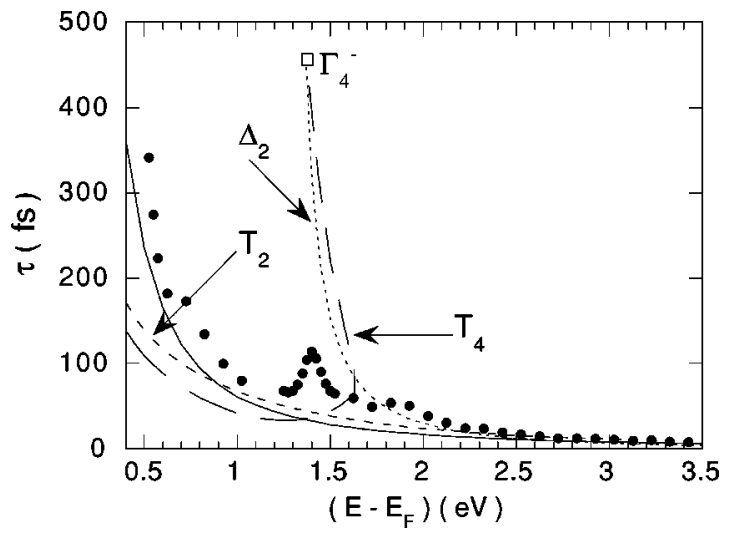

FIG. 7. Hot-electron lifetimes in Be. Solid circles and the solid line represent the same quantities as in Fig. 6. Short-dashed and long-dashed lines represent the lifetime of hot electrons in bands $T_{2}$ and $T_{4}$, respectively, with the wave vector along the $\Gamma K$ direction. The dotted line represents the lifetime of hot electrons in the $\Delta_{2}$ band along the $\Gamma A$ direction. The open square is located at the energy of the level $\Gamma_{4}^{-}$, showing the beginning and the end of the $\Delta_{2}$ and $T_{4}$ bands, respectively.

At the $\Gamma$ point, the free-electron parabola opens a wide energy gap around the Fermi level and splits along the $\Gamma K$ and $\Gamma M$ directions into two bands, $T_{2} / T_{4}$ and $\Sigma_{1} / \Sigma_{3}$, respectively. The results of our calculated lifetimes of hot electrons in bands $T_{2}$ and $T_{4}$, with the wave vector along the $\Gamma K$ direction, are plotted in Fig. 7 by short-dashed and longdashed lines, respectively. For comparison, the average lifetime of hot electrons in real beryllium and in a FEG with $r_{s}=1.87$ are also plotted in this figure by solid circles and by a solid line, respectively. At very-low electron energies ( $E$ $-E_{F}<1 \mathrm{eV}$ ), interband transitions yield lifetimes of hot electrons in the $T_{2}$ band that are below those of electrons in a FEG, as in the case of $\mathrm{Al}$ and $\mathrm{Mg}$. However, at higher energies the coupling with lower-lying flat bands becomes small, and lifetimes of electrons in this $\left(T_{2}\right)$ band are found to be above the FEG prediction. Lifetimes of hot electrons in the $T_{4}$ band are also, at very-low electron energies $\left(E-E_{F}\right.$ $<1.4 \mathrm{eV}$ ), below those of electrons in a FEG. Nevertheless, at energies of $\sim 1.4 \mathrm{eV}$ the presence of the band gap at $\Gamma$ yields very long lifetimes, especially at the level $\Gamma_{4}^{-}$.

We have calculated hot-electron lifetimes in bands $\Sigma_{1}$ and $\Sigma_{3}$, with the wave vector along the $\Gamma M$ direction, and have found results that are similar to those obtained for electrons in bands $T_{2}$ and $T_{4}$. Calculations of the lifetime of hot electrons in the $\Delta_{2}$ band, with the wave vector along the $\Gamma A$ direction, are also shown in Fig. 7 (dotted line). Though this is not a flat band, the presence of the gap at the $\Gamma$ point near the Fermi level results in hot electrons close to the level $\Gamma_{4}^{-}$ living much longer than in the case of a FEG. The combined contribution from hot electrons in bands $T_{4}, \Sigma_{1}$, and $\Delta_{2}$ is the origin of the enhanced average lifetime (solid circles) at $E-E_{F} \sim 1.4 \mathrm{eV}$, which corresponds to the energy of the level $\Gamma_{4}^{-}$at the $\Gamma$ point.

A wide band gap is also opened at the $L$ point, which originates the enhanced average lifetime at $E-E_{F}$ $\sim 1.8 \mathrm{eV}$. Hence, we have plotted in Fig. 8 the results of our calculated lifetimes of hot electrons in the $S_{1}$ band, with the wave vector along the $L H$ direction (dotted line), together

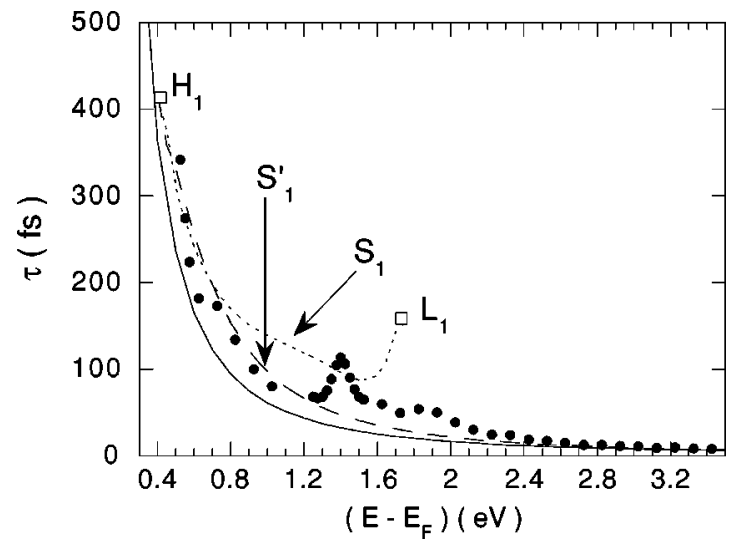

FIG. 8. Hot-electron lifetimes in Be. Solid circles and the solid line represent the same quantities as in Fig. 6. Dotted and dashed lines represent the lifetime of hot electrons in bands $S_{1}$ and $S_{1}^{\prime}$, respectively, with the wave vector along the $L H$ and $H A$ directions. Open squares are located at the energy of the levels $L_{1}$ and $H_{1}$.

with the average lifetime of hot electrons in real Be (solid circles) and in a FEG with $r_{s}=1.87$ (solid line). As in the case of hot electrons in bands $T_{4}$ and $\Sigma_{1}$, the presence of the band gap at the $L$ point yields a very long average lifetime, but now at $E-E_{F} \sim 1.8 \mathrm{eV}$. A similar behavior is obtained near the $L$ point for electrons in the $R_{1} R_{3}$ band along the $L A$ direction, and both bands $S_{1}$ and $R_{1} R_{3}$ contribute to the enhanced average lifetime at $E-E_{F} \sim 1.8 \mathrm{eV}$. In Fig. 8 we have also represented calculations of the lifetime of hot electrons in the $S_{1}^{\prime}$ band along the $H A$ direction (dashed line). At low energies $\left(E-E_{F}<2 \mathrm{eV}\right)$, the presence of the gap at the $H$ point leads to hot-electron lifetimes along the $H A$ direction that are longer than those of electrons in a FEG, but departure from free-electron behavior at the $H$ point is not as pronounced as at the $\Gamma$ or $L$ points. At higher energies, the $S_{1}^{\prime}$ band shows great similarity with the corresponding hcp free-electron band, and lifetimes nearly coincide, therefore, with those obtained within the FEG model.

\section{Copper}

Copper, the most widely studied metal by TR-2PPE, is a noble metal with entirely filled $3 d$-like bands. In Fig. 9 we show the energy bands of this fcc crystal. We see a profound difference between the band structure of $\mathrm{Cu}$ and that of free

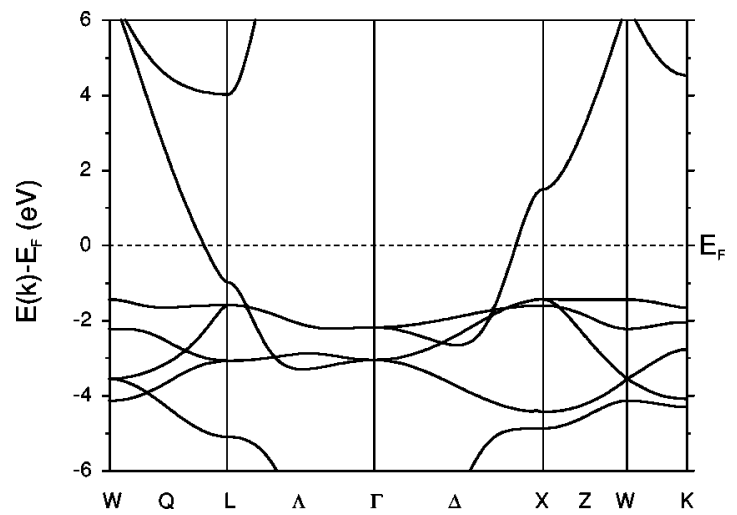

FIG. 9. Calculated band structure of $\mathrm{Cu}$ along certain symmetry directions. 


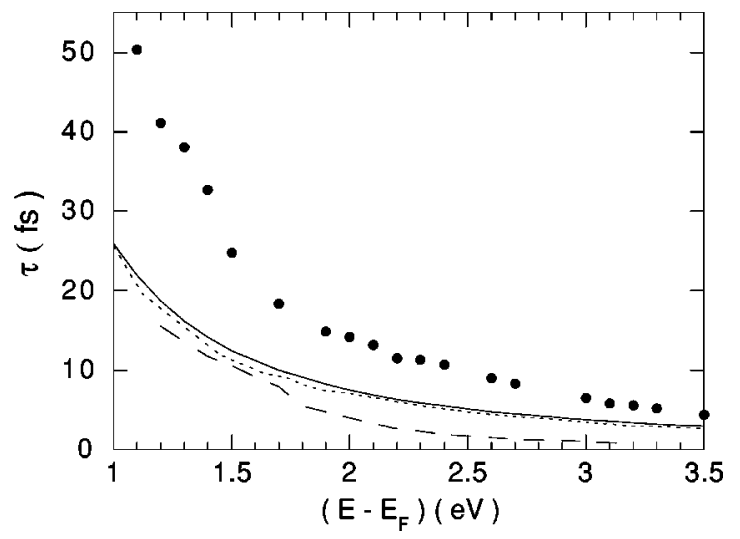

FIG. 10. Hot-electron lifetimes in $\mathrm{Cu}$ as in Fig. 2 with $r_{s}$ $=2.67$. The dashed line represents the result of replacing all oneelectron Bloch states by plane waves (FEG calculation) but keeping in Eq. (12) the actual number of states available for real transitions (Ref. 46). The dotted line represents our full ab initio calculation of $\tau(E)$, as obtained after averaging $\tau\left(\mathbf{k}, n_{i}\right)$ of Eq. (9) over wave vectors and over the band structure for each $\mathbf{k}$, but with the $3 d$ shell assigned to the core in the pseudopotential generation.

electrons. Slightly below the Fermi level, at $E-E_{F} \sim 2 \mathrm{eV}$, we have $d$ bands capable of holding 10 electrons per atom, the one remaining electron being in a free-electron-like band below and above the $d$ bands. Hence, a combined description of both delocalized $4 s^{1}$ and localized $3 d^{10}$ electrons is needed to address the actual electronic response of this material. The results presented below have been found by keeping all $4 s^{1}$ and $3 d^{10}$ Bloch states as valence electrons in the pseudopotential generation.

Band-structure $G W$-RPA calculations of the average lifetime $\tau(E)$ of hot electrons in $\mathrm{Cu}$ are exhibited in Fig. 10 by solid circles, as obtained from Eq. (9) with full inclusion of crystalline local-field effects. The lifetime of hot electrons in a FEG with the electron density equal to that of $4 s^{1}$ electrons in $\mathrm{Cu}\left(r_{s}=2.67\right)$ is represented by a solid line. These calculations indicate that the lifetime of hot electrons in $\mathrm{Cu}$ is, within RPA, larger than that of electrons in a FEG with $r_{s}$ $=2.67$, this enhancement varying from a factor of $\sim 2.5$ near the Fermi level $\left(E-E_{F}=1.0 \mathrm{eV}\right)$ to a factor of $\sim 1.5$ for $E$ $-E_{F}=3.5 \mathrm{eV}$. In order to investigate the role that localized $d$ bands play in the decay mechanism of hot electrons, we have also used an $a b$ initio pseudopotential with the $3 d$ shell assigned to the core. The result of this calculation, displayed in Fig. 10 by a dotted line, shows that it nearly coincides with the FEG calculation; thus, $d$-band states play a key role in the hot-electron decay.

We have performed band-structure calculations of Eq. (9) with and without [see also Eq. (11)] the inclusion of crystalline local-field corrections, and have found that these corrections are negligible for $E-E_{F}>1.5 \mathrm{eV}$, while for energies very near the Fermi level, neglecting these corrections results in an overestimation of the lifetime of less than $5 \%$. Therefore, differences between our full band-structure calculations (solid circles) and FEG calculations (solid line) come from the actual density of states (DOS) available for real excitations, localization, additional screening, and Fermi-surface topology.
First of all, we focus on the role that both DOS and coupling between Bloch states participating in the creation of $e-h$ pairs, i.e., localization [see Eq. (12)] play in the hotelectron decay mechanism. Hence, we neglect crystalline local-field effects and present the result of evaluating hotelectron lifetimes from Eq. (11) by replacing initial and final states in $\left|B_{i f}(\mathbf{q}+\mathbf{G})\right|^{2}$ by plane waves and the dielectric function in $\left|\epsilon_{\mathbf{G}, \mathbf{G}}(\mathbf{q}, \omega)\right|^{-2}$ by that of a FEG with $r_{s}=2.67$. If we further replaced $\operatorname{Im}\left[\epsilon_{\mathbf{G}, \mathbf{G}}(\mathbf{q}, \omega)\right]$ by that of a FEG, then we would obtain the FEG calculation represented by a solid line. The impact of the actual DOS below the Fermi level may be described by simply replacing the one-electron Bloch states in Eq. (12) by plane waves but keeping the actual number of states available for real excitations. The result of this calculation ${ }^{46}$ is represented in Fig. 10 by a dashed line. This result is very close to that reported by Ogawa et al.,$^{13}$ though these authors approximated the FEG dielectric function in $\left|\epsilon_{\mathbf{G}, \mathbf{G}}(\mathbf{q}, \omega)\right|^{-2}$ within the static Thomas-Fermi model.

It is clear from Fig. 10 that the actual DOS available for real transitions yields lifetimes that are shorter than those obtained in a FEG model, especially for $E-E_{F}>2 \mathrm{eV}$ due to opening of the $d$-band scattering channel dominating the DOS with energies $\sim 2 \mathrm{eV}$. However, if one takes into account, within a full description of the band structure of the crystal in the evaluation of $\operatorname{Im}\left[\epsilon_{\mathbf{G}, \mathbf{G}}(\mathbf{q}, \omega)\right]$, the actual coupling between initial and final states avaliable for real transitions, then one obtains hot-electron lifetimes which lie, at very-low electron energies $\left(E-E_{F}<2.5 \mathrm{eV}\right)$, just above the FEG curve (see open circles in Fig. 1 of Ref. 21). This enhancement of the lifetime, even at energies below the opening of the $d$-band scattering channel, is due to the fact that states just below the Fermi level have a small but significant $d$ component, thus being more localized than pure $s p$ states.

The combined effect of DOS and localization, which enters through the imaginary part of the dielectric matrix $\operatorname{Im}\left[\epsilon_{\mathbf{G}, \mathbf{G}^{\prime}}(\mathbf{q}, \omega)\right]$, increases the lifetime of hot electrons with energies $E-E_{F}<2.5 \mathrm{eV}$ (see open circles in Fig. 1 of Ref. 21). As for the departure of hot-electron initial and final states from free-electron behavior, entering through the coefficients $B_{i f}(\mathbf{q}+\mathbf{G})$, we have found that it yields hotelectron lifetimes that are strongly directional dependent, with Fermi surface shape effects tending to decrease the average inelastic lifetime of very-low-energy electrons ( $E$ $-E_{F}<2.5 \mathrm{eV}$ ) (see Ref. 21). Furthermore, the combined effect of DOS and localization, on the one hand, and Fermisurface shape effects, on the other hand, nearly compensate. Consequently, large differences between hot-electron lifetimes in real $\mathrm{Cu}$ and in a FEG with $r_{s}=2.67$, are mainly due to a major contribution from $d$ electrons participating in the screening of electron-electron interactions, which is accounted for by the factor $\left|\epsilon_{\mathbf{G}, \mathbf{G}}(\mathbf{q}, \omega)\right|^{-2}$ in Eq. (11).

The Fermi surface of $\mathrm{Cu}$ is greatly flattened in certain regions, showing a pronounced neck in the direction $\Gamma L$. Thus, the isotropy of hot-electron lifetimes in a FEG disappears in this material. While flattening of the Fermi surface along the $\Gamma K$ direction is found to decrease the hot-electron lifetime by a factor that varies from $\sim 15 \%$ near the Fermi level $\left(E-E_{F}=1 \mathrm{eV}\right)$ to $\sim 5 \%$ for $E-E_{F}=3.5 \mathrm{eV}$ (see also Ref. 47), the lifetime of hot electrons with the wave vector along the necks of the Fermi surface, in the $\Gamma L$ direction, is 
found to be much longer than the average lifetime. We have calculated hot-electron lifetimes in the $\Lambda_{1}$ band, with the wave vector along the $\Gamma L$ direction, and have found the lifetime of hot electrons at the $L_{1}$ level with $E-E_{F}=4.2 \mathrm{eV}$ to be longer than the average lifetime at this energy by $\sim 80 \%$.

A comparison between our calculated hot-electron lifetimes in $\mathrm{Cu}$ and those determined from most recent TR-2PPE experiments was presented in Ref. 21. At $E-E_{F}<2 \mathrm{eV}$, our calculations are close to lifetimes recently measured by Knoesel et al. in the very-low energy range. ${ }^{16}$ At larger electron energies, good agreement between our band-structure calculations and experiment is obtained for $\mathrm{Cu}(110),{ }^{13}$ the only surface with no band gap in the $\mathbf{k}_{\|}=0$ direction.

\section{CONCLUSIONS}

We have presented full $G W$-RPA band-structure calculations of the inelastic lifetime of hot electrons in $\mathrm{Al}, \mathrm{Mg}, \mathrm{Be}$, and $\mathrm{Cu}$, and have demonstrated that decay rates of lowenergy excited electrons strongly depend on the details of the electronic band structure. Though the dependence of hotelectron lifetimes in $\mathrm{Al}$ and $\mathrm{Mg}$ on the direction of the wave vector has been found not to be large, in the case of Be and $\mathrm{Cu}$ hot-electron lifetimes have been found to be strongly directional dependent. Furthermore, very long lifetimes at certain points of the $\mathrm{BZ}$ in Be yield average lifetimes in this material that strongly deviate from the $\sim\left(E-E_{F}\right)^{-2}$ scaling predicted within Fermi-liquid theory.

As far as band-structure effects on hot-electron energies and wave functions are concerned, we have found that both splitting of the band structure and the presence of band gaps over the Fermi level play an important role in the $e-e$ decay mechanism. In $\mathrm{Al}$ and $\mathrm{Mg}$, splitting of the band structure is found to yield electron lifetimes that are smaller than those of electrons in a FEG. On the other hand, large deviations of the band structure of Be along certain symmetry directions from the free-electron model near the Fermi level result in a strong directional dependence of hot-electron lifetimes in this material.

As for the presence of band-structure effects on the creation of $e-h$ pairs, there are contributions from the actual DOS available for real transitions, from localization, i.e., the actual coupling between electron and hole states, and from screening. The combined effect of DOS and localization is found not to be large, even in the case of a noble metal like $\mathrm{Cu}$ with $d$ bands. However, large differences between hotelectron lifetimes in $\mathrm{Cu}$ and in a FEG with the electron density equal to that of valence $\left(4 s^{1}\right)$ electrons are found to be due to a major contribution from $d$ electrons participating in the screening of $e-e$ interactions.

Crystalline local-field corrections in these materials have been found to be small for hot-electron energies under study.

\section{ACKNOWLEDGMENTS}

We would like to thank A. G. Eguiluz for stimulating discussions. We also acknowledge partial support by the University of the Basque Country, the Basque Hezkuntza, Unibertistate eta Ikerketa Saila, and the Spanish Ministerio de Educación y Cultura.
${ }^{1}$ J. J. Quinn and R. A. Ferrell, Phys. Rev. 112, 812 (1958).

${ }^{2}$ R. H. Ritchie, Phys. Rev. 114, 644 (1959).

${ }^{3}$ J. J. Quinn, Phys. Rev. 126, 1453 (1962).

${ }^{4}$ R. H. Ritchie and J. C. Ashley, J. Phys. Chem. Solids 26, 1689 (1965).

${ }^{5}$ L. Kleinman, Phys. Rev. B 3, 2982 (1971).

${ }^{6}$ J. C. Shelton, Surf. Sci. 44, 305 (1974).

${ }^{7}$ D. R. Penn, Phys. Rev. B 13, 5248 (1976).

${ }^{8}$ C. J. Tung, J. C. Ashley, and R. H. Ritchie, Surf. Sci. 81, 427 (1979).

${ }^{9}$ D. R. Penn, Phys. Rev. B 22, 2677 (1980).

${ }^{10}$ D. R. Penn, Phys. Rev. B 35, 482 (1987).

${ }^{11}$ C. A. Schmutenmaer, M. Aeschlimann, H. E. Elsayed-Ali, R. J. D. Miller, D. A. Mantell, J. Cao, and Y. Gao, Phys. Rev. B 50, 8957 (1994).

${ }^{12}$ T. Hertel, E. Knoesel, M. Wolf, and G. Ertl, Phys. Rev. Lett. 76, 535 (1996).

${ }^{13}$ S. Ogawa, H. Nagano, and H. Petek, Phys. Rev. B 55, 1 (1997).

${ }^{14}$ J. Cao, Y. Gao, R. J. D. Miller, H. E. Elsayed-Ali, and D. A. Mantell, Phys. Rev. B 56, 1099 (1997).

${ }^{15}$ M. Aeschlimann, M. Bauer, S. Pawlik, W. Weber, R. Burgermeister, D. Oberli, and H. C. Siegmann, Phys. Rev. Lett. 79, 5158 (1997).

${ }^{16}$ E. Knoesel, A. Hotzel, and M. Wolf, Phys. Rev. B 57, 12812 (1998).

${ }^{17}$ A. Goldmann, R. Matzdorf, and F. Theilmann, Surf. Sci. 414, L932 (1998).
${ }^{18}$ J. Cao, Y. Gao, H. E. Elsayed-Ali, R. J. D. Miller, and D. A. Mantell, Phys. Rev. B 58, 10948 (1998).

${ }^{19}$ M. Bauer, S. Pawlik, and M. Aeschlimann, Proc. SPIE 3272, 201 (1998).

${ }^{20}$ H. Petek and S. Ogawa, Prog. Surf. Sci. 56, 239 (1998).

${ }^{21}$ I. Campillo, J. M. Pitarke, A. Rubio, E. Zarate, and P. M. Echenique, Phys. Rev. Lett. 83, 2230 (1999).

${ }^{22}$ W.-D. Schöne, R. Keyling, M. Bandić, and W. Ekardt, Phys. Rev. B 60, 8616 (1999).

${ }^{23}$ P. Hohenberg and W. Kohn, Phys. Rev. 136, B864 (1964); W. Kohn and L. Sham, Phys. Rev. 140, A1133 (1965).

${ }^{24}$ A. L. Fetter and J. D. Walecka, Quantum Theory of ManyParticle Systems (McGraw-Hill, New York, 1971).

${ }^{25}$ L. Hedin and S. Lundqvist, Solid State Phys. 23, 1 (1969).

${ }^{26}$ E. Runge and E. K. U. Gross, Phys. Rev. Lett. 52, 997 (1984).

${ }^{27}$ M. Petersilka, U. J. Gossmann, and E. K. U. Gross, Phys. Rev. Lett. 76, 1212 (1996).

${ }^{28}$ P. M. Echenique, J. M. Pitarke, E. V. Chulkov, and A. Rubio, Chem. Phys. 251, 1 (2000).

${ }^{29}$ The so-called electron-density parameter $r_{s}$ is defined by the relation $1 / n_{0}=(4 / 3) \pi r_{s}^{3}, n_{0}$ being the average electron density.

${ }^{30}$ V. M. Silkin, E. V. Chulkov, I. Yu. Sklyadneva, and V. E. Panin, Izv. Vuzov. Fiz. 9, 56 (1984) [Sov. Phys. J. 27, 762 (1984)]; E. V. Chulkov, V. M. Silkin, and E. N. Shirykalov, Fiz. Met. Metalloved. 64, 213 (1987) [Phys. Met. Metallogr. 64, 1 (1987)].

${ }^{31}$ N. Troullier and J. L. Martins, Phys. Rev. B 43, 1993 (1991). 
${ }^{32}$ J. P. Perdew and A. Zunger, Phys. Rev. B 23, 5048 (1981).

${ }^{33}$ D. M. Ceperley and B. J. Alder, Phys. Rev. Lett. 45, 1196 (1980).

${ }^{34}$ D. J. Singh, Plane Waves, Pseudopotentials, and the LAPW Method (Kluwer, Boston, 1994).

${ }^{35}$ I. Campillo, A. Rubio, and J. M. Pitarke, Phys. Rev. B 59, 12188 (1999).

${ }^{36}$ H. J. Monkhorst and J. D. Pack, Phys. Rev. B 13, 5188 (1976).

${ }^{37}$ P. M. Platzman, E. D. Isaacs, H. Williams, P. Zschack, and G. E. Ice, Phys. Rev. B 46, 12943 (1992).

${ }^{38}$ W. Schulke, H. Schulte-Schrepping, and J. R. Schmitz, Phys. Rev. B 47, 12426 (1993).

${ }^{39}$ A. Fleszar, A. A. Quong, and A. G. Eguiluz, Phys. Rev. Lett. 74, 590 (1995).

${ }^{40}$ B. C. Larson, J. Z. Tischler, E. D. Isaacs, P. Zschack, A. Fleszar, and A. G. Eguiluz, Phys. Rev. Lett. 77, 1346 (1997).
${ }^{41}$ I. Campillo, J. M. Pitarke, and A. G. Eguiluz, Phys. Rev. B 58, 10 307 (1998).

${ }^{42}$ The prediction of Eq. (13) (dashed line in the inset of Figs. 2, 4, 6, and 10) would coincide, as $E \rightarrow E_{F}$, with the full RPA free-electron gas calculation only in the high-density $\left(r_{s} \rightarrow 0\right)$ limit.

${ }^{43}$ S. P. Singhal and J. Callaway, Phys. Rev. B 16, 1744 (1977).

${ }^{44}$ F. Szumulowicz and B. Segall, Phys. Rev. B 21, 5628 (1980).

${ }^{45}$ D. A. Papaconstantopoulos, Handbook of the Band Structure of Elemental Solids (Plenum Press, New York, 1986).

${ }^{46}$ We have followed Ogawa et al. (Ref. 13) to take the electrondensity parameter $r_{s}$ in the evaluation of $\left|\epsilon_{\mathbf{G}, \mathbf{G}}(\mathbf{q}, \omega)\right|^{-2}$ from the actual DOS at the Fermi level.

${ }^{47}$ S. L. Adler, Phys. Rev. 130, 1654 (1963). 